\section{Mortalidad general e infantil en Chile en el largo plazo, 1909-2017}

\author{
MANUEL LLORCA-JAÑA ${ }^{1, a, c}$, RODRIGO RIVERO-CANTILLANO ${ }^{2, b, c}$, \\ JAVIER RIVAS ${ }^{1, b, d}$, MARTINA ALLENDE ${ }^{1, b}$
}

\section{General and infant mortality trends in Chile from 1909 to 2017}

Background: During the twentieth century, Chile experienced an important reduction in general mortality. Aim: To describe both general and infant mortality of Chile from 1909 to 2017. Material and Methods: Analysis of information about births and deaths published by the Chilean National Institute of Statistics for the period between 1909 and 2017. Results: Both general and infant mortality rates declined sharply from the 1930s to the late 1990s. However, during the last few years, general mortality rates increased slightly. This is the first increase in over a century. Another positive aspect is that there was a dramatic decrease in mortality rate gaps across Chilean regions, for both general and infant mortality. However, intraregional inequalities in infant mortality continue to be a detrimental factor. Conclusions: Public health efforts should be carried out to further reduce socioeconomic and regional gaps in adult and infant mortality in Chile.

(Rev Med Chile 2021; 149: 1047-1057)

Key words: Epidemiology: Demography; Infant Mortality; Mortality.
'Escuela de Administración Pública,

Universidad de Valparaíso. Valparaíso,

Chile.

${ }^{2}$ Facultad de Artes Liberales, Universidad

Adolfo Ibáñez. Santiago, Chile.

aEconomista.

${ }^{b}$ Historiador(a).

‘PhD en Historia Económica.

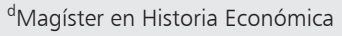

Financiamiento: Proyecto Anillos, ANID PIA SOC180001 y Fondecyt Regular

1210144. La ANID no tuvo influencia en el diseño del estudio; ni en la recolección, análisis o interpretación de los datos; ni en la preparación, revisión o aprobación del manuscrito. Estamos muy agradecidos de los comentarios de dos revisores, por su exhaustiva lectura.

Recibido el 2 de octubre de 2020, aceptado el 8 de mayo de 2021.

Correspondencia a: Manuel Llorca-Jaña.

Universidad de Valparaíso, Las Heras 6, Valparaíso, Chile. manuel.Ilorca@uv.cl
$\mathrm{E}$ n el siglo XX Chile experimentó una caída abrupta de su tasa de mortalidad general, en particular a partir de la década de 1930-39 y hasta fines de la de 1990-99 (Figura 1). Las décadas 1930-1960 pertenecen al período de industrialización dirigida por el Estado (cuando emergió un estado de bienestar caracterizado por mayor gasto en salud y educación), mientras que desde mediados de los años 1970-79 el país se encuentra bajo un modelo económico neoliberal. La caída en la mortalidad es parte integral de la transición demográfica y epidemiológica ocurrida en nuestro país ${ }^{1}$. Como parte de este proceso, la mortalidad infantil, en particular, descendió de manera acelerada desde mediados de la década de 1930-39 hasta fines de los años 1990-99². Estos hechos constituyen los principales acontecimientos en la historia demográfica del país, y en general de la mayoría de las naciones desarrolladas y de ingreso medio durante el último siglo. Lo anterior reviste gran importancia, toda vez que la tasa de mortalidad es uno de los principales indicadores de desarrollo económico y de condiciones y niveles de salud de una comunidad ${ }^{3-5}$, así como de desigualdad ${ }^{6}$. Esto es particularmente cierto en países como Chile, que registran buenas estadísticas vitales para el siglo XX, en particular a partir de mediados de siglo, cuando se expandió de manera significativa la cobertura de las oficinas del Registro Civil ${ }^{7}$.

A su vez, la caída en la mortalidad es uno de los elementos centrales de lo que se ha denominado como evolución tecnofisiológica de la humanidad, caracterizada por un aumento en la estatura, el peso y la esperanza de vida de las personas, gracias a la sinergia que ha existido entre avances tecnológicos y fisiológicos ${ }^{8}$. Chile no ha sido excepción. En efecto, la estatura promedio de la población chilena experimentó un importante aumento en 
el siglo $\mathrm{XX}^{9}$, mientras que el decrecimiento en la tasa de mortalidad se ha traducido en un aumento notorio de la esperanza de vida al nacer ${ }^{10}$, toda vez que la mayor caída en la tasa de mortalidad se ha producido en menores de un año ${ }^{5}$.

La caída en la mortalidad general en Chile ha atraído la atención de diversos autores ${ }^{1,2,4,11-16}$, existiendo, además, estudios específicos que dan cuenta del declive en la mortalidad infantil ${ }^{6,12,13,17-27}$. Sin embargo, las publicaciones existentes se centran en períodos relativamente acotados de tiempo, que abarcan a lo sumo 3-4 décadas, sin entregar una visión de conjunto que abarque la totalidad del siglo XX y primeras décadas del XXI, salvo el trabajo de Rodrigo, van der Veer, Vermeer y van Ijzendoorn ${ }^{28}$, que cubre algunos aspectos de la mortalidad infantil. Este es particularmente el caso para análisis de mortalidad a nivel regional. Asimismo, el período anterior a 1950 ha despertado poca atención, salvo por unos pocos trabajos ${ }^{29}$. Producto de lo anterior, subsisten tendencias de largo plazo que no han sido identificadas, o no han recibido suficiente atención. Asimismo, la evolución de las diferencias de mortalidad a nivel regional no ha sido analizada en profundidad para el largo plazo, salvo algunas excepciones ${ }^{4,30}$. Con todo, el propósito de este estudio es entregar una visión de largo plazo de la evolución de la mortalidad general e infantil en Chile en el último siglo, así como identificar algunos de los principales hechos que las han caracterizado, enfatizando en particular en la evolución de la mortalidad infantil, tanto a nivel nacional como regional.

\section{Material y Método}

Para este estudio hemos empleado los anuarios de estadísticas vitales, o demográficos, del Instituto Nacional de Estadísticas (INE), en cuanto a población, nacimientos y defunciones se refiere. En particular, hemos ingresado en Stata y Excel las siguientes variables, para cada año, entre 1909 y 2017: número de defunciones totales, número de defunciones por género, población, número de defunciones por rango etario, número de nacimientos, orden de nacimiento de los nacidos vivos, y defunción de menores de un año según orden de nacimiento. Posteriormente, para obtener series de defunciones por rangos etarios fijos, así como para simplificar la información entregada en algunas de las figuras, hemos reagrupado los datos. Finalmente, cabe destacar que las estadísticas vitales de 2004 se encuentran incompletas para algunas de estas variables. Además de la información a nivel nacional, para mortalidad general e infantil, ingresamos datos a nivel provincial para estas variables, así como para población y nacimientos. Posteriormente reagrupamos los datos a nivel provincial en siete grandes regiones. Sobre la base de la división político-administrativa vigente, las siete grandes regiones se agrupan de la siguiente manera. Norte Grande: regiones de Arica y Parinacota, Tarapacá y Antofagasta; Norte Chico: Regiones de Atacama y Coquimbo; Centro Norte: regiones de Valparaíso y O’Higgins; Centro Sur: Región de Maule; Sur: regiones de Bío-Bío, Araucanía, Los Ríos y Los Lagos; Austral: regiones de Aysén y Magallanes; y, por último, la Región Metropolitana.

\section{Resultados}

\section{Tendencias generales y por género}

La tasa de mortalidad general en Chile cayó sistemáticamente desde comienzos de siglo XX hasta principios de los años 2000 (Tabla 1), pasando desde un promedio anual para la década de 1910-19 de 30,4 defunciones por mil habitantes hasta 5,3 por $1.000 \mathrm{~h}$ en la primera década del siglo XXI. Sin embargo, en los últimos años dicha tasa ha crecido levemente, promediando 5,7 para los últimos 8 años disponibles (2010-2017), tal como ha ocurrido en muchos otros países. No obstante, lo anterior, actualmente la tasa de mortalidad de Chile se encuentra muy por debajo del promedio mundial (7,7 por $1.000 \mathrm{~h}$ para 2015$)$, y levemente por debajo del latinoamericano (5,9 por $1.000 \mathrm{~h}$ para 2015) ${ }^{31}$. Las décadas de mayor caída porcentual, respecto de la década anterior, fueron las décadas de 1950-59, 1970-79, 1940-49 y 1980-89, en orden descendente. Separando los datos por género, la tasa de mortalidad de mujeres es menor a la de hombres (Figura 1), como ocurre en el resto del mundo ${ }^{3,4}$. Sin embargo, el ratio de mortalidad de hombres a mortalidad de mujeres ha fluctuado de manera significativa. El mismo aumentó desde 1,06 por $1.000 \mathrm{~h}$ por año en los períodos 1910-1920 hasta 1,28, en la década de los 1980, para luego experimentar una caída gradual desde la década siguiente hasta un 1,15 en la última década (2010). 
Tabla 1. Mortalidad en Chile, 1910-2017, promedios anuales según sexo, por década

\begin{tabular}{|cccccc|}
\hline Período & Hombres & $\begin{array}{c}\text { Tasa de mortalidad } \\
\text { Mujeres }\end{array}$ & $\begin{array}{c}\text { Ambos sexos } \\
\text { Amariación interdécada } \\
\text { para ambos sexos }\end{array}$ & $\begin{array}{c}\text { Ratio } \\
\text { Hombres/Mujeres }\end{array}$ \\
\hline $1910-9$ & 31,3 & 29,5 & 30,4 & & 1,06 \\
$1920-9$ & 28,7 & 27,1 & 27,9 & $-8,4 \%$ & 1,06 \\
$1930-9$ & 24,5 & 22,8 & 23,6 & $-15,3 \%$ & 1,07 \\
$1940-9$ & 19,3 & 17,6 & 18,5 & $-21,7 \%$ & 1,10 \\
\hline $1950-9$ & 14,1 & 12,1 & 13,1 & $-29,3 \%$ & 1,17 \\
\hline $1960-9$ & 11,9 & 9,7 & 10,8 & $-17,6 \%$ & 1,22 \\
\hline $1970-9$ & 8,6 & 6,8 & 7,7 & $-28,7 \%$ & 1,27 \\
\hline $1980-9$ & 6,8 & 5,3 & 6,1 & $-21,0 \%$ & 1,28 \\
\hline $1990-9$ & 6,1 & 4,9 & 5,5 & $-9,9 \%$ & 1,25 \\
\hline $2000-9$ & 5,8 & 4,8 & 5,3 & $-3,0 \%$ & 1,20 \\
\hline $2010-7$ & 6,1 & 5,3 & 5,7 & $7,2 \%$ & 1,15 \\
\hline
\end{tabular}

Nota: Defunciones por 1.000 habitantes. Se calculó un promedio simple de los valores anuales para cada década.

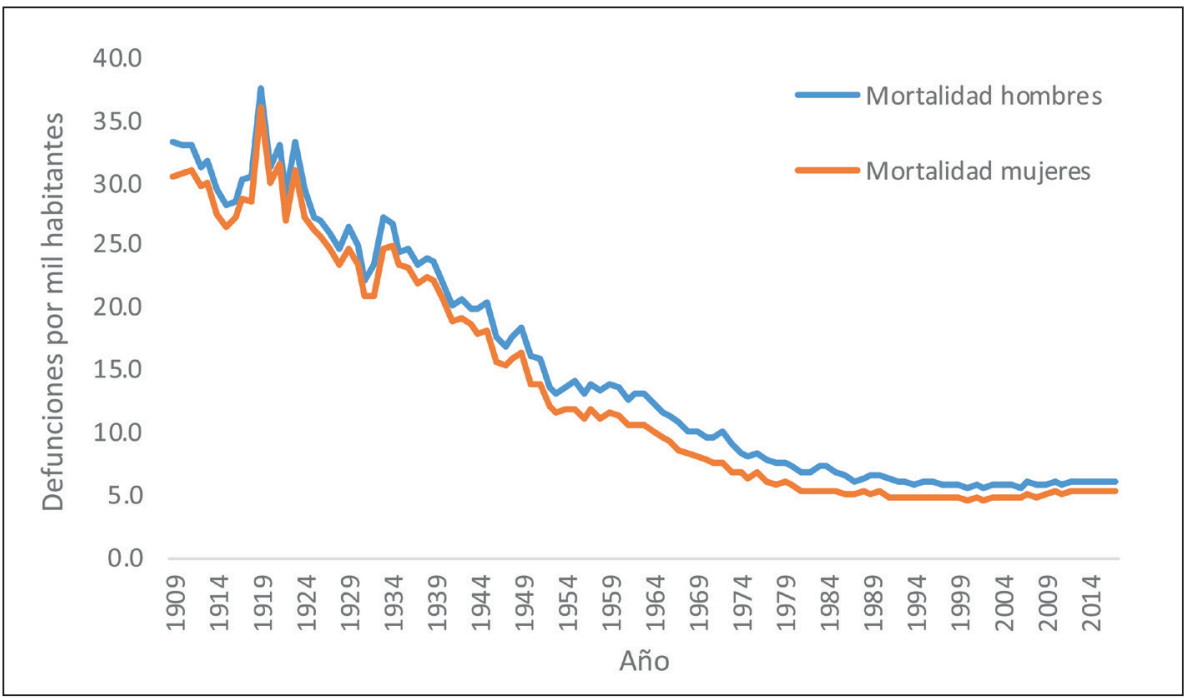

Figura 1. Mortalidad general por género, Chile, 1909-2017.

\section{Mortalidad general por regiones}

La Tabla 2 contiene la mortalidad de Chile desagregada por grandes regiones para 1920-2017. En la primera década para la que tenemos datos, la de 1920-29, había una gran dispersión territorial en la mortalidad general. La zona Centro-Sur era la que tenía una mayor tasa de mortalidad, seguida por la Región Metropolitana, registrando un notorio exceso de muertes respecto del promedio nacional. La zona Austral, en cambio, era la que tenía una menor tasa de mortalidad, un hecho ya destacado por Taucher ${ }^{13}$. A partir de la década de 1930-39 se observa un notable proceso de convergencia de todas las regiones, reduciéndose la desigualdad territorial ante la mortalidad. En efecto, desde la década 1980-89, las diferencias en las tasas de mortalidad por regiones son muy menores, con una desviación estándar inferior a 0,8 para el período 1980-2017. Cabe destacar que la misma convergencia se ha observado en las tasas de fecundidad ${ }^{32}$. 
Tabla 2. Mortalidad por grandes regiones de Chile, promedios anuales por década, 1920-2017

\begin{tabular}{|ccccccccc|}
\hline Década & $\begin{array}{c}\text { Norte } \\
\text { Grande }\end{array}$ & $\begin{array}{c}\text { Norte } \\
\text { Chico }\end{array}$ & $\begin{array}{c}\text { Centro } \\
\text { Norte }\end{array}$ & $\begin{array}{c}\text { Gran Región } \\
\text { Metro- } \\
\text { politana }\end{array}$ & $\begin{array}{c}\text { Centro } \\
\text { Sur }\end{array}$ & Sur & Austral & $\begin{array}{c}\text { Desviación } \\
\text { estándar }\end{array}$ \\
\hline $1920-9$ & 24,0 & 26,4 & 28,4 & 29,8 & 43,8 & 23,5 & 18,9 & 7,9 \\
\hline $1930-9$ & 19,8 & 23,2 & 22,8 & 24,0 & 43,3 & 21,2 & 16,1 & 8,8 \\
\hline $1940-9$ & 15,9 & 16,8 & 17,8 & 16,8 & 31,7 & 17,2 & 13,2 & 6,0 \\
\hline $1950-9$ & 12,5 & 14,3 & 13,4 & 11,5 & 24,7 & 13,7 & 10,9 & 4,7 \\
\hline $1960-9$ & 10,3 & 11,4 & 11,0 & 9,8 & 20,4 & 11,6 & 9,2 & 3,8 \\
\hline $1970-9$ & 7,7 & 7,6 & 8,4 & 7,1 & 12,6 & 8,5 & 7,4 & 1,9 \\
\hline $1980-9$ & 5,3 & 5,6 & 6,5 & 5,6 & 7,0 & 6,9 & 5,6 & 0,7 \\
\hline $1990-9$ & 4,9 & 5,0 & 6,0 & 5,2 & 6,2 & 6,2 & 5,4 & 0,6 \\
\hline $2000-9$ & 4,7 & 5,0 & 6,1 & 5,1 & 6,0 & 6,0 & 5,7 & 0,6 \\
\hline $2010-7$ & 5,0 & 5,6 & 6,5 & 5,6 & 6,7 & 6,3 & 5,9 & 0,6 \\
\hline
\end{tabular}

Nota: Defunciones por 1.000 habitantes. Se calculó un promedio simple de los valores anuales para cada década.

\section{Composición de la mortalidad general según rango etario}

La Tabla 3 y la Figura 2 muestran la proporción de defunciones por grupos de edad para el período 1932-2017. La Tabla 3 contiene información para un mayor número de rangos que la Figura 2. De los mismos se desprende que ha existido una transformación substancial en la composición de la mortalidad según edad del fallecido/a. Hasta fines de la década de 1960-69, más de 30\% del total de defunciones en Chile correspondía a menores de un año, cifra que llega a más de $40 \%$ si consideramos todos los menores de 10 años. A partir de fines de la década 1960-69, se produce una caída acelerada en la mortalidad infantil. En la última década, en promedio anual, solo 2,2\% de los fallecidos tienen menos de 10 años. Como contraparte, $71 \%$ de las defunciones corresponde a mayores de 64 años. La participación de este último grupo etario ha ido creciendo sostenidamente, desde tan solo $15 \%$ en 1930-39, conforme aumenta su proporción en la estructura etaria de la población.

Tabla 3. Composición porcentual de las defunciones según rango etario, Chile, 1932-2017

\begin{tabular}{|c|c|c|c|c|c|c|c|c|c|c|c|}
\hline \multirow[t]{2}{*}{ Período } & \multicolumn{11}{|c|}{ Edad de fallecimiento (en años) } \\
\hline & $\begin{array}{c}\text { Menor } \\
\text { a } 1\end{array}$ & $1-4$ & 5-9 & $10-14$ & $15-19$ & 20-29 & 30-39 & $40-49$ & $50-59$ & 60-64 & $\begin{array}{l}65 \text { y } \\
\text { más }\end{array}$ \\
\hline $1932-9$ & $33,4 \%$ & $13,2 \%$ & $2,6 \%$ & $1,7 \%$ & $3,1 \%$ & $7,8 \%$ & $6,7 \%$ & $6,4 \%$ & $6,5 \%$ & $3,5 \%$ & $14,7 \%$ \\
\hline $1940-9$ & $31,7 \%$ & $11,7 \%$ & $2,2 \%$ & $1,8 \%$ & $3,2 \%$ & $7,2 \%$ & $6,7 \%$ & $6,7 \%$ & $7,0 \%$ & $4,4 \%$ & $16,9 \%$ \\
\hline $1950-9$ & $32,6 \%$ & $8,6 \%$ & $1,7 \%$ & $1,2 \%$ & $1,7 \%$ & $4,9 \%$ & $5,4 \%$ & $6,8 \%$ & $8,5 \%$ & $5,2 \%$ & $23,1 \%$ \\
\hline $1960-9$ & $31,9 \%$ & $6,4 \%$ & $1,4 \%$ & $0,9 \%$ & $1,4 \%$ & $3,8 \%$ & $5,3 \%$ & $6,7 \%$ & $9,3 \%$ & $5,9 \%$ & $26,8 \%$ \\
\hline $1970-9$ & $19,0 \%$ & $3,0 \%$ & $1,2 \%$ & $1,1 \%$ & $1,6 \%$ & $3,9 \%$ & $4,8 \%$ & $7,5 \%$ & $10,4 \%$ & $7,1 \%$ & $40,5 \%$ \\
\hline $1980-9$ & $8,2 \%$ & $1,5 \%$ & $0,7 \%$ & $0,7 \%$ & $1,2 \%$ & $3,9 \%$ & $4,4 \%$ & $6,7 \%$ & $11,5 \%$ & $7,5 \%$ & $53,9 \%$ \\
\hline $1990-9$ & $4,5 \%$ & $0,9 \%$ & $0,5 \%$ & $0,5 \%$ & $1,0 \%$ & $3,2 \%$ & $4,2 \%$ & $6,1 \%$ & $10,0 \%$ & $7,6 \%$ & $61,5 \%$ \\
\hline $2000-9$ & $2,4 \%$ & $0,4 \%$ & $0,3 \%$ & $0,3 \%$ & $0,8 \%$ & $2,4 \%$ & $3,5 \%$ & $6,0 \%$ & $9,8 \%$ & $7,2 \%$ & $66,8 \%$ \\
\hline 2010-7 & $1,7 \%$ & $0,3 \%$ & $0,2 \%$ & $0,2 \%$ & $0,6 \%$ & $2,0 \%$ & $2,5 \%$ & $5,0 \%$ & $9,8 \%$ & $6,9 \%$ & $70,8 \%$ \\
\hline
\end{tabular}




\section{Tendencias generales de la mortalidad infantil}

Dado que el cambio más importante en la composición de la mortalidad según grupos de edad es la caída tan substancial que experimentó la mortalidad del grupo de menores de un año, la Figura 3 y la Tabla 4 muestran la evolución de la mortalidad infantil en Chile. La tasa de mortalidad infantil comenzó a declinar con fuerza desde mediados de la década de 1930-39 (cuando se registraban alrededor de 250 muertes de menores de un año por cada 1.000 nacidos vivos), de manera acelerada e ininterrumpida, salvo por la segunda mitad de la década de 1950-59, cuando se estancó cerca de un lustro, fenómeno que se observó simultáneamente en varios países ${ }^{29}$. En la actualidad se encuentra en torno a 7 defunciones por cada mil nacidos/as vivos/as, menos de un cuarto del promedio mundial, que alcanzó a 33 en el 2015, y menos de la mitad del promedio latinoamericano ${ }^{17}$ ese mismo año, alcanzando en la actualidad el ratio más bajo dentro de Sudamérica ${ }^{31}$. Respecto de los promedios anuales por década, las caídas porcentuales más significativas se dieron en las décadas de 1980-89, 1990-99 y 1970-79, en ese orden de magnitud.

\section{Mortalidad infantil, algunos hechos adicionales destacados}

Una proporción creciente de los nacimientos en Chile corresponden al primero o segundo hijo de sus madres. La Figura 4 muestra el orden de nacimiento (paridad) desde 1930. En la década de $1930-39,48 \%$ de los nacidos vivos eran primero o segundo hijo. En la última década (2010) esta tasa aumentó a 77\%. En contraste, aquellos nacidos que eran cuarto hijo/a de la madre, o más allá del cuarto, representaban $38 \%$ de los nacidos en 1930-39, pero tan solo $7 \%$ en 2010-17. No existen cifras de mortalidad infantil según orden de nacimiento de los fallecidos, pero la Figura 5 contiene el ratio "defunción de menores de un año según orden de nacimiento"/“nacidos vivos según mismo orden de nacimiento". Como puede apreciarse, este ratio es sistemáticamente mayor para niños/niñas que fueron el cuarto hijo/a o un orden superior a 4. Finalmente, entre 1920 y $1980-89$, la mortalidad postneonatal fue siempre mayor que la neonatal.

\section{Mortalidad infantil por regiones}

La Tabla 5 contiene la mortalidad infantil de Chile desagregada por regiones para 1920-2017. Tal como para la mortalidad general, se observa un notable proceso de convergencia en la tasa de mortalidad infantil entre las regiones chilenas. En las últimas décadas las diferencias son muy menores. Dicho proceso se ha atribuido en particular a la expansión en la cobertura de agua potable y alcantarillado, así como a la universalización de servicios de salud ${ }^{17}$.

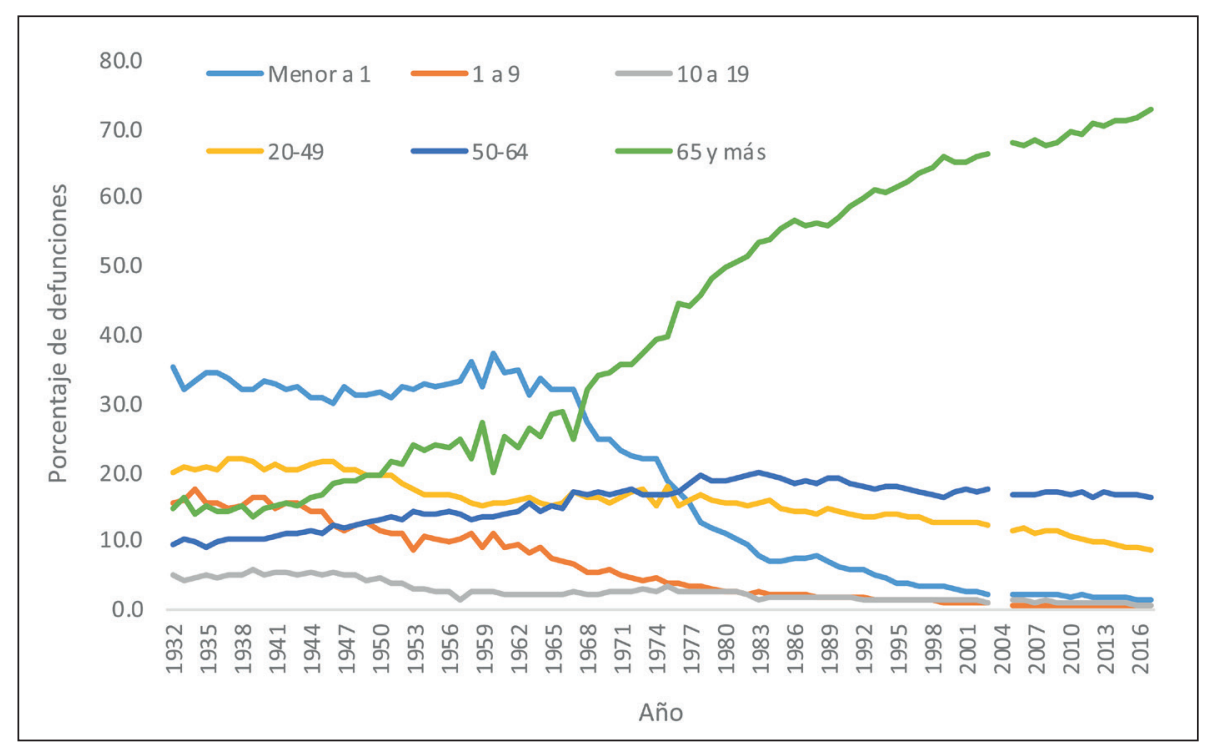

Figura 2. Porcentaje de defunciones según rango etario (años), Chile, 1932-2017. 


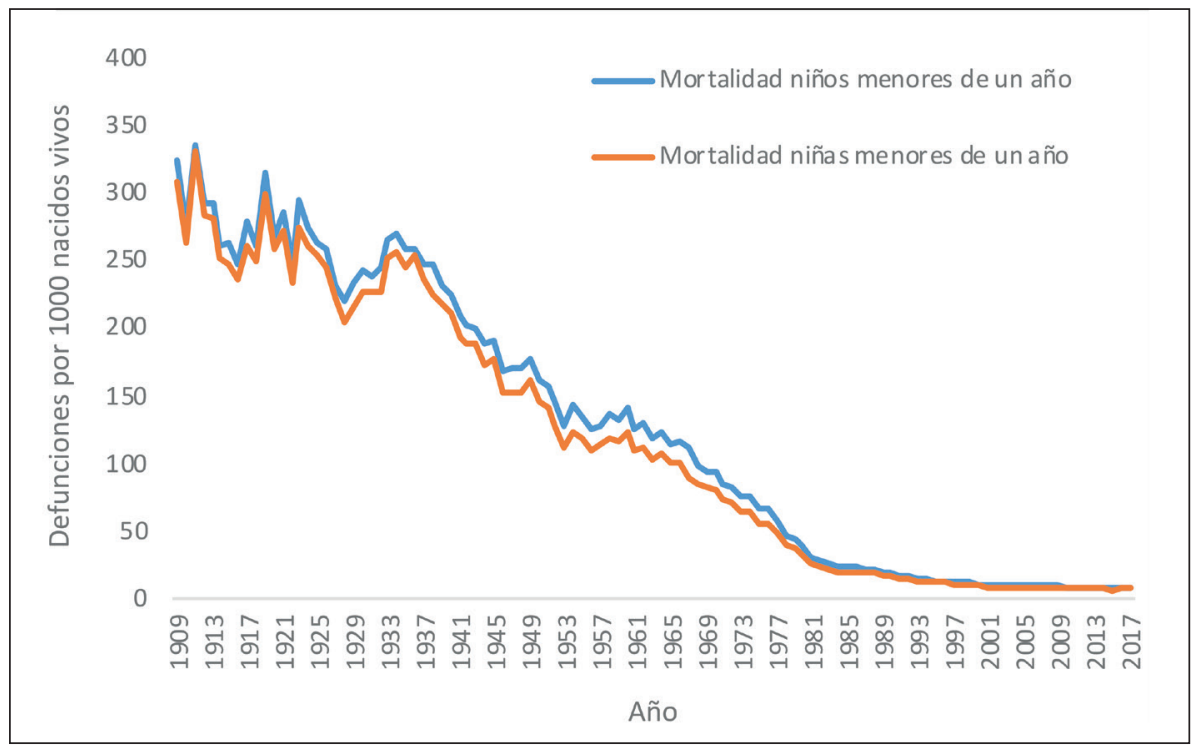

Figura 3. Mortalidad infantil por sexo, Chile, 1909-2017.

Tabla 4. Mortalidad infantil, Chile, promedios anuales por década, 1915-2017

\begin{tabular}{|ccccc|}
\hline Período & $\begin{array}{c}\text { Mortalidad infantil } \\
\text { (menores de un año) }\end{array}$ & $\begin{array}{c}\text { Variación } \\
\text { interdécada }\end{array}$ & $\begin{array}{c}\text { Mortalidad } \\
\text { postneonatal }\end{array}$ & $\begin{array}{c}\text { Mortalidad } \\
\text { neonatal }\end{array}$ \\
\hline $1915-19$ & 265,2 & 124,8 & 140,4 \\
\hline $1920 s$ & 250,2 & $-6 \%$ & 129,4 & 120,8 \\
\hline $1930 s$ & 242,9 & $-3 \%$ & 136,9 & 106,0 \\
\hline $1940 s$ & 182,1 & $-25 \%$ & 112,1 & 70,0 \\
\hline $1950 s$ & 130,2 & $-29 \%$ & 86,2 & 36,0 \\
\hline $1960 s$ & 108,8 & $-16 \%$ & 72,0 & 26,5 \\
\hline $1970 s$ & 63,4 & $-42 \%$ & 36,9 & 11,0 \\
\hline $1980 s$ & 23,0 & $-64 \%$ & 12,0 & 7,2 \\
\hline $1990 s$ & 12,9 & $-44 \%$ & 5,7 & 5,7 \\
\hline $2000 s$ & 8,4 & $-35 \%$ & 2,7 & 5,3 \\
\hline $2010 s$ & 7,2 & $-14 \%$ & 1,9 & \\
\hline
\end{tabular}

\section{Discusión}

Durante los últimos 120 años, Chile ha experimentado una notable mejoría en dos indicadores fundamentales de desarrollo económico y de condiciones y niveles de salud: mortalidad general y mortalidad infantil, lo que se ha traducido en disfrutar una de las mayores esperanzas de vida de la región. Asimismo, se ha observado una notoria disminución de la desigualdad regional para estos dos indicadores de mortalidad. Este fenómeno es loable, toda vez que reducir la desigualdad regional 

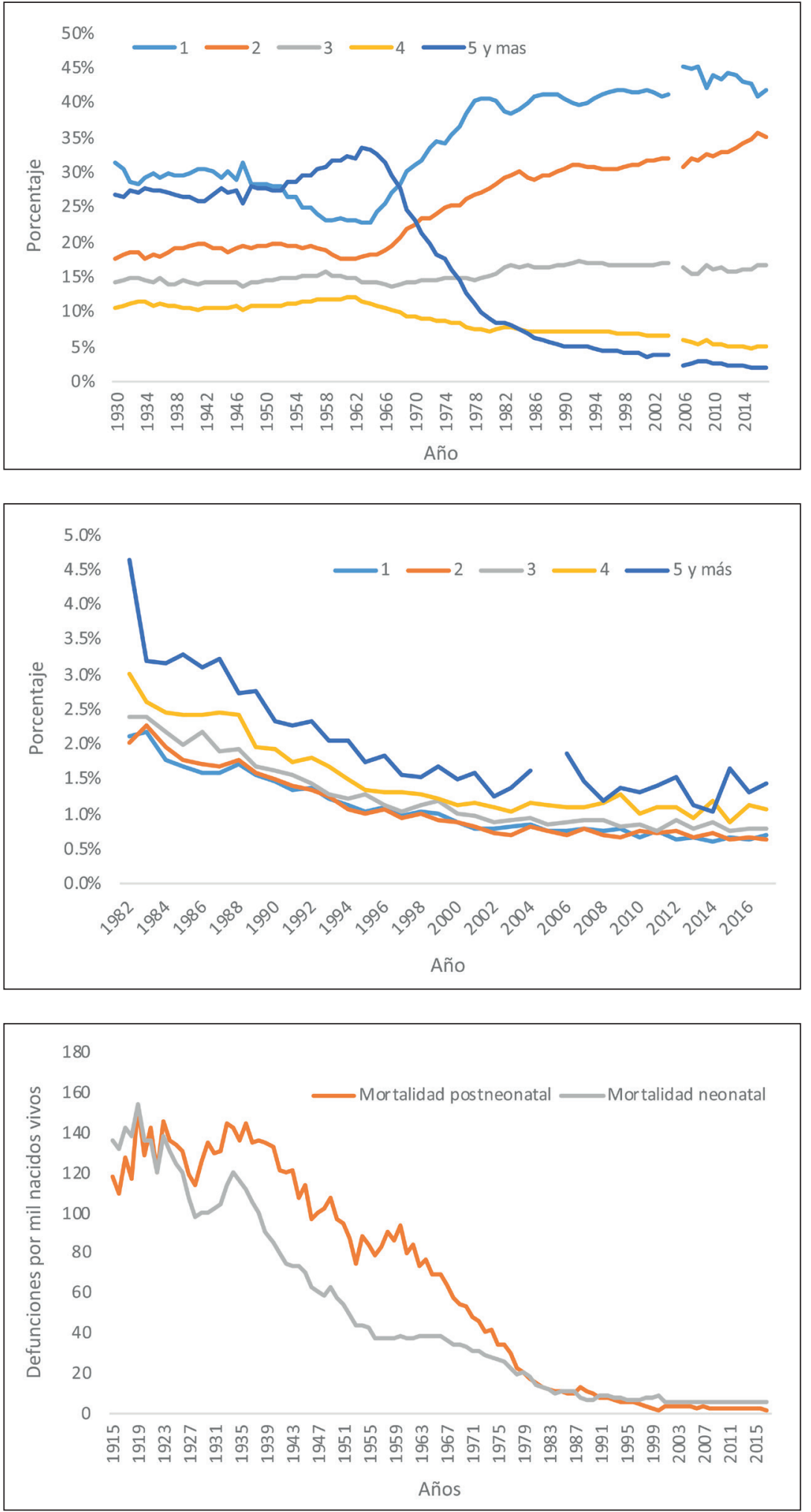

Figura 4. Nacidos vivos según orden de nacimiento (paridad), 1930-2017.

Figura 5. Ratio "defunción de menores de un año según orden de nacimiento" / "nacidos vivos según mismo orden de nacimiento".

Figura 6. Mortalidad infantil, neonatal y postneonatal, Chile, 1915-2017. 
Tabla 5. Mortalidad infantil por regiones, promedios anuales por década, Chile, 1920-2017

\begin{tabular}{|ccccccccc|}
\hline Década & $\begin{array}{c}\text { Norte } \\
\text { Grande }\end{array}$ & $\begin{array}{c}\text { Norte } \\
\text { Chico }\end{array}$ & $\begin{array}{c}\text { Centro } \\
\text { Norte }\end{array}$ & $\begin{array}{c}\text { Gran Región } \\
\text { Metro- } \\
\text { politana }\end{array}$ & $\begin{array}{c}\text { Centro } \\
\text { Sur }\end{array}$ & Sur & Austral & $\begin{array}{c}\text { Desviación } \\
\text { estándar }\end{array}$ \\
\hline $1920-9$ & 203,2 & 203,5 & 232,6 & 279,0 & 265,0 & 249,1 & 146,5 & 45,2 \\
\hline $1930-9$ & 189,7 & 196,9 & 219,9 & 229,6 & 289,2 & 269,2 & 146,8 & 48,5 \\
\hline $1940-9$ & 142,8 & 165,2 & 165,6 & 153,3 & 206,2 & 214,2 & 118,4 & 33,9 \\
\hline $1950-9$ & 115,8 & 132,5 & 120,8 & 97,4 & 147,1 & 163,1 & 97,3 & 24,6 \\
\hline $1960-9$ & 98,0 & 113,5 & 94,7 & 77,5 & 126,9 & 150,4 & 89,3 & 25,0 \\
\hline $1970-9$ & 53,6 & 67,9 & 55,5 & 42,3 & 73,6 & 92,1 & 61,9 & 16,1 \\
\hline $1980-9$ & 21,8 & 26,5 & 21,7 & 17,2 & 26,2 & 31,1 & 22,6 & 4,5 \\
\hline $1990-9$ & 12,9 & 14,3 & 12,4 & 11,3 & 13,7 & 15,3 & 12,9 & 1,3 \\
\hline $2000-9$ & 8,4 & 9,6 & 8,4 & 7,7 & 8,5 & 9,2 & 9,5 & 0,7 \\
\hline $2010-7$ & 7,4 & 7,7 & 7,0 & 6,9 & 7,2 & 7,7 & 7,5 & 0,3 \\
\hline
\end{tabular}

en indicadores de salud pública es por sí mismo un objetivo de salud pública ${ }^{30}$. A nivel sudamericano, el país ocupa el cuarto lugar en mortalidad general, y tiene la tasa más baja de mortalidad infantil. Sin embargo, en el caso de la mortalidad general, en los últimos años (2010-2017) la misma creció $7 \%$ respecto de la década previa, siendo este el primer aumento en más de cien años, lo que requiere especial atención de las autoridades sanitarias, aunque justo es decir que esta tendencia se explica, en parte, por el cambio de la estructura de edades de la población chilena, marcada por un proceso de envejecimiento rápido ${ }^{34}$. En efecto, si calculáramos la tasa de mortalidad ajustada por edad, la mortalidad general ha seguido bajando. Asimismo, a pesar de la disminución en la desigualdad territorial, persisten desigualdades ante la muerte al interior de las provincias ${ }^{35}$.

Las causas de la caída en la tasa de mortalidad general en Chile antes de 2010 son bien conocidas: adhesión irrestricta a la teoría de los gérmenes; caída en enfermedades infecciosas como causa de muerte, principalmente gracias al uso de antibióticos, sulfamidas, vacunación masiva y otras mejoras en atención médica que se vieron favorecidas con la creación del Servicio Nacional de Salud en 1952 (y la del Ministerio de Salud en
1924); caída en tasas de desnutrición y pobreza; expansión en provisión de servicios de electricidad, agua potable y alcantarillado; mejoras en educación e ingreso per cápita; y expansión de la cobertura de atención médica ${ }^{2,11,13,15,17,29,33,36}$. Debemos considerar también el hecho de partir de tasas de mortalidad infantil altas. De hecho, reducciones tan sustanciales en la mortalidad general son solo posibles en países con tasas de mortalidad infantil tan altas como las que tenía Chile ${ }^{8}$ y donde, a su vez, se implementen políticas adecuadas, como las observadas en Chile. El aumento de la mortalidad en los últimos años que hemos señalado se ha debido principalmente al proceso de envejecimiento demográfico, dinámica asociada mundialmente a las etapas más avanzadas de la transición demográfica ${ }^{37}$. Por otro lado, sobre la base de la transición epidemiológica, ha aumentado la mortalidad por cáncer, enfermedades asociadas al tabaquismo y al alcoholismo, así como por enfermedades ligadas a la hipertensión arterial, diabetes mellitus, sobrepeso y obesidad ${ }^{1,2,33}$. A pesar de este aspecto negativo, un aspecto positivo ha sido la gran convergencia en tasas de mortalidad para todas las regiones de Chile, lo que da cuenta de riesgos actuales similares de muerte independientemente del 
lugar de nacimiento/residencia de la población, cuestión que no ocurría durante la primera mitad del siglo XX.

Respecto de la mortalidad infantil, a principios del siglo XX, Chile tenía una de las más altas tasas a nivel mundial ${ }^{38}$. La abrupta caída de la misma en pocas décadas es un gran logro que debe destacarse, siendo la mejora más espectacular en toda la región ${ }^{39}$, y una de las cinco más importantes a nivel mundial entre 1960 y $2010^{40}$. Esta revolución en la mortalidad infantil se debió principalmente a los siguientes factores: campañas de vacunación de amplia cobertura, mayor cobertura de servicios de salud, hasta casi alcanzar la universalización; disminución de la desnutrición infantil; focalización del gasto en salud en grupos de menores ingresos, y en particular en mujeres embarazadas e infantes, sobre todo en períodos de crisis económicas; programas de entrega de alimentos (leche en particular) a embarazadas, madres amamantando e infantes; caída en la fertilidad de forma tal que los ingresos familiares se distribuyen en un número menor de hijos; uso creciente de refrigeradores y cocinas en hogares; mejor nutrición de la población en general; mejor educación de madres y padres, incluyendo en hábitos alimentarios; aumento de la urbanización y consiguiente aumento de acceso a mejores servicios de salud, agua potable y servicios de alcantarillado (así como disminución del tamaño de las familias); caída en muertes causadas por ciertas enfermedades como diarrea y neumonía; progresos en el tratamiento de condiciones complejas en las etapas neonatal y obstétricas, especialmente desde los años 199099 14,25-28,30,39,41-44. Aun cuando la tasa de mortalidad infantil no ha dejado de caer, a pesar de ser la más baja de Sudamérica, esta tasa se encuentra aún distante de la de muchos países desarrollados, varios de los cuales gozan de tasas de mortalidad infantil por debajo de 3 defunciones por mil nacidos vivos (algunos incluso por debajo de 2, como Finlandia e Islandia). Vale decir, hay espacio para seguir mejorando en este indicador, realizando esfuerzos adicionales en materia de salud pública y de distribución del ingreso ${ }^{28}$.

Asimismo, en los últimos años ha habido un leve estancamiento de la tasa de mortalidad infantil. Varios trabajos ${ }^{7,18,22,34,45}$ han atribuido este fenómeno a la desigualdad imperante en el país: grupos socioeconómicos de menores ingresos enfrentan un riesgo mayor de mortalidad infantil. Tanto
Jiménez y Romero ${ }^{6}$ como Hertel-Fernández, Giusti y Sotelo ${ }^{22}$ han hecho un llamado a reforzar las intervenciones de salud pública en sectores de menores ingresos, para poder seguir reduciendo dicha tasa, y converger a la que disfrutan algunos países desarrollados. Esto incluye mayores y mejores intervenciones antenatales, neonatales y postnatales, aunque justo es destacar la caída en la tasa de mortalidad neonatal hasta mediados de la década 1990-99. En virtud de la actual desigualdad imperante en salud, que explica parte del estallido social de octubre 2019, dichas recomendaciones adquieren aun mayor relevancia, con el fin último de disminuir la desigualdad social ante la muerte.

Agradecimientos: Los autores agradecen los comentarios de los revisores, por su exhaustiva lectura.

\section{Referencias}

1. Szot J. La transición demográfico-epidemiológica en Chile, 1960- 2001. Rev Esp Salud Pública 2003; 77 (5): 605-13.

2. Medina E, Kaempffer A. Tendencias y características de la mortalidad chilena 1970-2003. Rev Med Chile 2007; 135: 240-50.

3. Sen A. Mortality as an indicator of economic success and failure. The Econ J. January 1998; 108 (446): 1-25.

4. Medina E, Kaempffer A. Mortalidad del adulto en Chile. Rev Med Chile 2000; 128: 1144-9.

5. CEPAL. La mortalidad en América Latina: una trayectoria auspiciosa pero heterogénea. América Latina y el Caribe Observatorio Demográfico: Mortalidad. Octubre 2007; 2 (4): 9-43.

6. Jiménez J, Romero MI. Reducing infant mortality in Chile: success in two phases. Health Affairs. 2007: 26 (2): 458-65.

7. Behm H, Correa M. La mortalidad en los primeros años de vida en países de la América Latina: Chile 1965 - 1966. San José: Celade 1977.

8. Fogel RW. The Escape from Hunger and Premature Death, 1700-2100. New York: Cambridge University Press 2004.

9. Llorca-Jaña M, Navarrete-Montalvo J, Araya R, Droller F, Allende M, Rivas J. Height in twentieth-century Chilean men: growth with divergence. Cliometrica 2020.

10. Wagner G, Díaz J, Lüders R. Chile 1810-2010. La República en cifras. Santiago: Ediciones UC 2016.

11. Zepeda A, Monteverde L. Compresión de la mortalidad 
en Chile: 1969-2002. Papeles Poblac. 2016; 87: 265-91.

12. Taucher E. Chile, mortalidad desde 1955 a 1975: tendencias y causas. Santiago: CELADE 1978.

13. Taucher E. Mortalidad infantil en Chile. Tendencias, diferenciales y causas. Santiago: CELADE 1979.

14. Taucher E, Albala C, lcaza G. Adult Mortality from Chronic Discases in Chile, 1968-90. In: Timaeus J, Chackicl J, Ruzicka L. editors. Adult Mortality in Latin America. Oxford: Claredon Press 1996; 266-8.

15. Chackiel J. La dinámica demográfica en América Latina. CEPAL: Santiago 2004.

16. Chackiel J, Plaut R. América Latina. Tendencias demográficas con énfasis en la mortalidad Notas de Población 1994; 22 (60): 11-45.

17. Castañeda T. Contexto socioeconómico y causas del descenso de la mortalidad infantil en Chile. Documento de Trabajo no 28. Santiago: Centro de Estudios Públicos (CEP) 1984.

18. Szot J. Mortalidad infantil e indicadores económicos en Chile 1985-1999. Rev Med Chile 2002; 130: 107-12.

19. Taucher E, Jofre I. Mortalidad infantil en Chile: El gran descenso. Rev Med Chile 1997; 125: 1225-35.

20. Behm H, et al. (1970). Mortalidad infantil en Chile: Tendencias recientes. Cuad Med Soc (Santiago) 1970; 11 (3): 55-67.

21. Bocaz A. Mortalidad infantil: procedimiento para el análisis de niveles, tendencias y diferenciales (caso de estudio: Chile, período 1937-1976). Santiago: CELADE 1980.

22. Hertel-Fernández A, Giusti A, Sotelo J. The Chilean infant mortality decline: improvement for whom? Socioeconomic and geographic inequalities in infant mortality, 1990-2005. Bull. World Health Organ. 2007; 85 (10): 798-804.

23. Hollstein RD, Vega J, Carvajal YB. Desigualdades sociales y salud. Nivel socioeconómico y mortalidad infantil en Chile, 1985-1995. Rev Med Chile 1998; 126: 333-40.

24. Raczynski D. Contexto socioeconómico del descenso de la mortalidad infantil en Chile. Estudios Públicos 1984; 16: 57-71.

25. Raczynski D, Oyarzo C. ¿Por qué cae la tasa de mortalidad infantil en Chile? Colección de Estudios CIEPLAN diciembre 1981; 6: 45-84.

26. Monckeberg F, Valiente, S, Mardones. Infant and PreSchool Nutrition: Economical Development, Versus Intervention Strategies. The Case of Chile. Nutr Res. 1987; 7 (3): 327-42.

27. Kaempffer AM, Medina E. La salud infantil en Chile durante la década del setenta. Rev Chil Pediatr. 1982; 53 (5): 468-80

28. Rodrigo A, Van Der Veer R, Vermeer H, Van Ijzendoorn
RH. From foundling homes to day care: a historical review of childcare in Chile. Cad. Saude Publica. 2014; 30 (3): 461-72.

29. Behm H. Recent Mortality Trends in Chile. Washington: Vital and Health Statistics, Serie 3, No 2, National Center for Health Statistics, U.S. Department of Health and Welfare; 1964.

30. Brieba D. State Capacity and Health Outcomes: Comparing Argentina's and Chile's Reductionof Infant and Maternal Mortality, 1960-2013. World Dev. 2018; 101: 37-53.

31. UN. Department of Economic and Social Affairs, Population Division. World Mortality 2017 - Data Booklet (ST/ESA/SER.A/412) [citado el 16 de septiembre de 2020]. Available from: https://www.un.org/en/development/desa/population/publications/pdf/mortality/ World-Mortality-2017-Data-Booklet.pdf.

32. Martínez J. La transición demográfica y las diferencias sociales de la fecundidad y la mortalidad infantil en Chile. Santiago: Ministerio de Planificación y Cooperación, Fondo de Población de las Naciones Unidas 1998.

33. Solimano G, Mazzei M. ¿De qué mueren los chilenos hoy?: perspectivas para el largo plazo. Rev Med Chile 2007; 135: 932-8.

34. Rivero-Cantillano R, Spijker J. Del rejuvenecimiento al envejecimiento de la población ¿o viceversa?: Chile en el contexto de América Latina, 1950-2050. Papeles Poblac. 2015; 101: 127-15.

35. Sánchez H, Albala C. Desigualdades en salud: mortalidad del adulto en comunas del Gran Santiago. Rev Med Chile 2004; 132: 453-60.

36. Milla M, González D. Dinámica demográfica de Chile y América Latina: una visión a vuelo de pájaro. Revista de Sociología 2004; 18: 81-116.

37. Rivero-Cantillano R, Spijker J. Del rejuvenecimiento al envejecimiento de la población ¿̇o viceversa?: Chile en el contexto de América Latina, 1950-2050. Notas de Población 2015; 101: 127-56.

38. Deichler C. Historia y alimentación popular. Dos décadas de lucha médica contra la desnutrición en el Chile urbano, 1930-1950. Santiago: Ministerio de Salud de Chile 2016.

39. Borrescio-Higa F, Bozzoli CG, Droller F. Early life environment and adult height: the case of Chile. 2019; Econ Hum Biol. 33 (1): 134-43.

40. Mcguire JW. Wealth, Health, and Democracy in East Asia and Latin America. New York: Cambridge University Press 2010.

41. Rivero-Cantillano R. La duración de la vida activa en la fuerza de trabajo del Gran Santiago, 1960-2002. Perfiles Económicos 2017; 4(1): 119-55. 
Mortalidad en Chile en el largo plazo - M. Llorca-Jaña et al

42. Goldsmith J. Milk makes State: the extension and implementation of Chile's state milk programs, 1901-1971. Historia 2017; 50 (1): 79-104.

43. Zárate MS. Por la salud del cuerpo. En Zárate MS, editor. Por la salud del cuerpo: historia y políticas sanitarias en Chile. Santiago: Universidad Alberto
Hurtado 2008; 10-34.

44. Vio F, Kain J, Gray E. Nutritional surveillance: The case of Chile, Nutr Res. 1992; 12 (3): 321-35.

45. Donoso E. Desigualdad en mortalidad infantil entre las comunas de la provincia de Santiago. Rev Med Chile 2004; 132: 461-6.

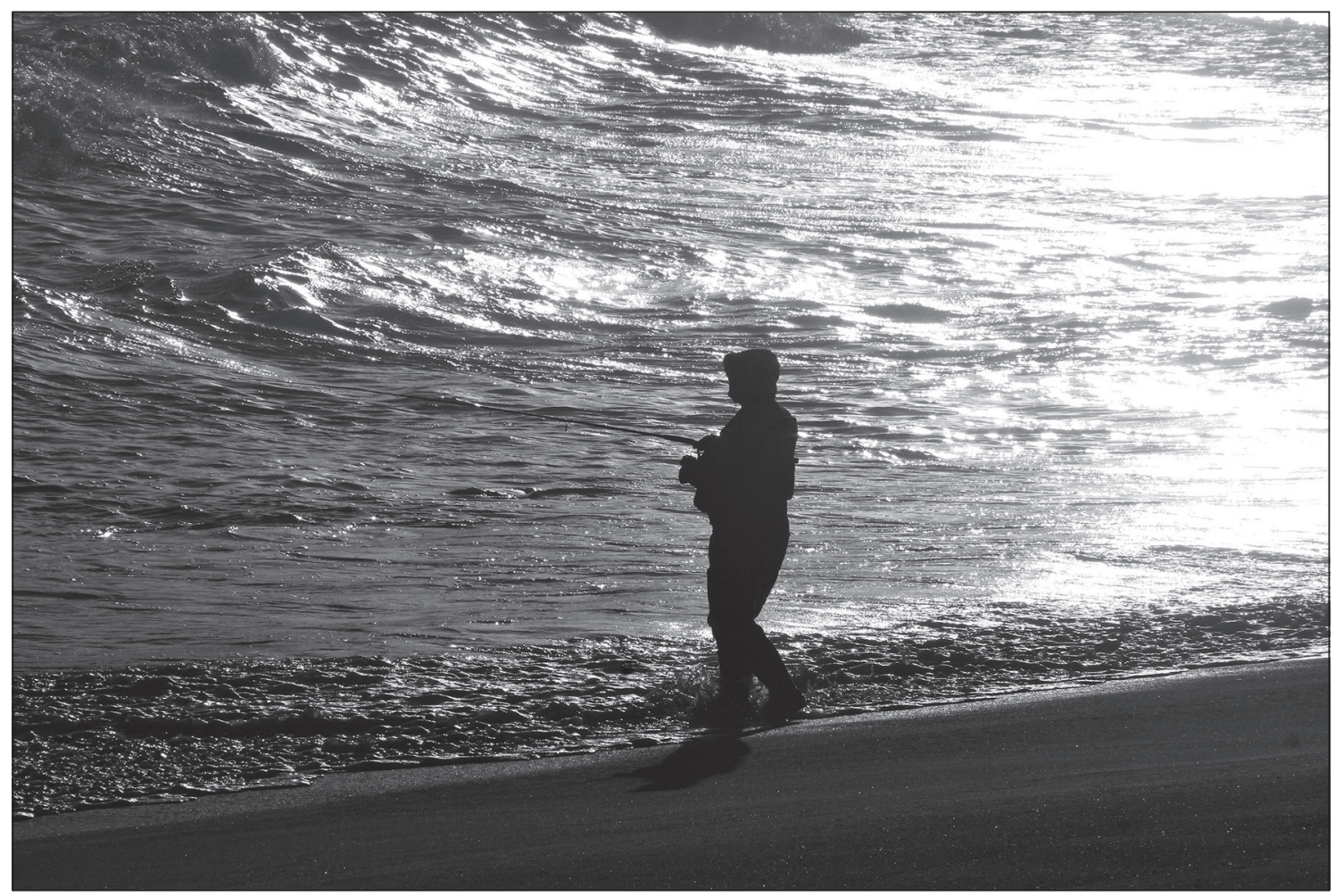

Playa Tunquén, Valparaíso. Dr. Jorge Sapunar Zenteno 